\title{
WRS/WARISATA
}

www.revistawarisata.org

Volumen 2 / Nro. 6 / Septiembre - diciembre 2020 / ISSN: 2708 - 6305 / ISSN-L: 2708 - 6305 / Pág. 156 - 175

\section{Modelo teórico para el desarrollo de competencias para la elaboración de arreglos musicales por parte de docentes en formación}

\author{
Theoretical model for the development of competences for the development of \\ musical arrangements by teachers in training
}

- Roberto Alexander Rodríguez Suárez

dr.profrobertorodriguez@gmail.com

Código ORCID: 0000-0001-5941-3705

Universidad Pedagógica Experimental Libertador, Venezuela
I Artículo recibido en junio 2020

I Arbitrado en julio 2020

I Publicado en septiembre 2020

\section{RESUMEN}

Palabras clave:

Educación Musical; competencias; mercado laboral; arreglo musical; modelo teórico
El objetivo de esta investigación fue generar un modelo teórico para el desarrollo de competencias relacionadas con el arreglo musical, que permita a los estudiantes de la especialidad Educación Musical de la Universidad Pedagógica Experimental Libertador, UPEL, Venezuela, un mejor desempeño durante su formación. La metodología fue abordada bajo el paradigma interpretativo y la teoría fundamentada. Se usó la entrevista a profundidad, la cual se aplicó a docentes y estudiantes de la especialidad, estudiantes de música y profesores no graduados que no son de música. Los resultados obtenidos permitieron construir el modelo teórico representado por una colmena, que a su vez, constituyeron los insumos para la formulación de las competencias para el arreglo musical. Con este modelo no solo se busca formar a un docente en el área de la música, sino también a un docente capaz de ser investigador, creativo, proactivo en todo lo que implica el quehacer docente.

\section{ABSTRACT}

The objective of this research was to generate a theoretical model for the development of competences related to the musical arrangement, which allows students of the specialty Musical Education of the Libertador Experimental Pedagogical University, UPEL, Venezuela, a better performance during their training. The methodology was approached under the interpretive paradigm and the grounded theory. The in-depth interview was used, which was applied to teachers and students of the specialty, music students and non-graduate professors who are not from music. The results obtained allowed the construction of the theoretical model represented by a beehive, which in turn constituted the inputs for the formulation of the competences for the musical arrangement. This model not only seeks to train a teacher in the area of music, but also a teacher capable of being a researcher, creative, proactive in everything that involves teaching. 


\section{INTRODUCCIÓN}

La música al igual que todas las disciplinas, es una profesión que requiere del desarrollo de habilidades, destrezas y conocimientos, que tienen cierto grado de conocimiento empírico y que exigen una gran dedicación.

A través de la historia, el empleo y el éxito profesional en el campo de la música han dependido, casi totalmente, de las competencias demostradas en las audiciones, recitales, conciertos, obras musicales, composiciones musicales, entre otros, por esta razón en el ámbito musical se convierte al maestro en la institución y no al maestro en parte la institución. De aquí proviene el hecho de preguntar: ¿Con quién estudias música?, en vez de preguntar: ¿Dónde estudias música? Por lo tanto, el estudio de la música, específicamente la armonía y la composición va a depender de con quien estudies, lo que convierte así a este maestro en la escuela de tu enfoque teórico para la composición y el arreglo musical.

La educación musical es una actividad que involucra diversos aspectos del desarrollo del ser humano. Esta comprende un conjunto de actividades que le permiten al individuo manejar su voz, agudizar la percepción auditiva, desarrollar su sentido rítmico natural y expresarse corporalmente mediante ella. Todas estas actividades están integradas de manera equitativa en un programa variado, significativo, interesante y diversificado de acuerdo con la edad y otras características de los individuos, esta se debe a que la música es el estímulo más apropiado para producir estados emocionales de diferente índole, para desarrollar la sensibilidad, desarrollar valores y actitudes constructivas, es el producto del intercambio social de los seres humanos, es considerada la expresión de la cultura en la sociedad, por eso es vista como el reflejo de las costumbres, tradiciones, religiones, ritos y bailes.

Los docentes en el área de educación musical deben enseñar a los alumnos de todas las edades habilidades para que estos puedan leer partituras (solfeo), tocar un instrumento o cantar. Estos dan clases en una amplia variedad de entornos, incluyendo escuelas, colegios, universidades y conservatorios (Guzmán y Rodríguez, 2012). Algunos maestros enseñan música en privado, en sus propias casas o en las de sus alumnos, poseen una amalgama de habilidades musicales, que les permiten desempeñarse en los diferentes roles del músico, como por ejemplo, dirigir agrupaciones musicales como: corales, estudiantinas, bandas escolares, bandas rítmicas, entre otras.

Los profesores de música son los responsables de dominar el lenguaje musical en su totalidad. Estos han adoptado como sus principales tareas el dominio de instrumentos musicales, de la técnica vocal y la integración de ambos aspectos; además, de estos apoyar como facilitadores en otras áreas del currículo básico, también deben ser músicos.

Los docentes especialistas en música deben leer, escribir y dominar la armonía musical que le permitirá plasmar toda su creatividad en los arreglos musicales. Las actividades extra cátedras referidas a asignaturas relacionadas con las artes, como el teatro, música, danza, selección de algún deporte, entre otras, como son consideradas en muchas instituciones educativas, son encargadas a los docentes que se preocupan por poner en escenario o en la cancha, según sea el caso, un producto donde se evidencie su labor como artista o deportista, dejando en último plano la labor educativa. De esta manera, el especialista siente que su trabajo no se queda estancado, y por otra parte estas 
actividades permiten realzar y promocionar la institución dentro y fuera del recinto educativo. Estas actividades exigen al docente especialista de música ir más allá del hecho de enseñar, es decir debe componer una canción y arreglar una obra, lo que implica tener otras habilidades y destrezas que no están incluidas en el currículo de la especialidad Educación Musical de la universidad, sin embargo, este puede tomar decisiones importantes para poder ejercer esa función y cumplir con los eventos que se planifican en las instituciones educativas.

Por tal motivo, la composición y el arreglo en la música debe estar más orientado hacia una función específica del docente especialista, por lo tanto, debe darse en las universidades como una especialización después de culminados los estudios de pregrado, sin embargo, en su formación de pregrado este debe desarrollar habilidades en esta área específica, para luego ser profundizada, debido a esto el especialista, en muchos casos, se le han exigido habilidades de un músico profesional, es decir, es como pretender que cada profesor de historia haga su libro o un profesor de castellano haga un poemario cada año.

Para componer y arreglar una obra musical hace falta una serie de conocimientos técnicos de la estructura de una obra musical, entre ellas; la armonía, la melodía, el ritmo, entre otras cosas, pero lo más importante para esto es tener una idea musical clara para arreglar una canción que sea agradable para el oyente. Por esta razón, se encuentran personas con ideas musicales, que les permiten hacer una obra o un arreglo sin tener el conocimiento técnico de la música, cosa que lo limita al performance de esta. Lo ideal es combinar lo académico con la experiencia del compositor o arreglista no perteneciente al sistema de educación musical formal.
En la Universidad Pedagógica Experimental Libertador (UPEL), específicamente en el seno de los cursos del componente especializado del programa de Educación Musical, el cual se administra en las sede de Rubio, Maracay, Barquisimeto, Caracas y Miranda, los estudiantes de la especialidad han manifestado que para poder cumplir con muchas de esas actividades deben arreglar o componer una canción para los diferentes eventos que organiza el Ministerio del Poder Popular para la Educación, la Zona Educativa, el Distrito Escolar, las escuelas públicas y privadas donde laboran, y otros entes similares. Además es necesario un mayor número de semestres por curso o varios niveles para cada materia, con un mayor número de horas, para poder obtener una formación más completa y sólida, con el fin de no necesitar acudir a una escuela de música o conservatorio para ampliar los conocimientos adquiridos en dicha institución.

En la actualidad, el mercado laboral requiere de un personal con habilidades digitales que no son desarrolladas en la universidad, como a esta situación no escapan los docentes, los estudiantes de la especialidad Educación Musical de la UPEL, necesitan desarrollar aún más habilidades ligadas al oficio del especialista en música que al del docente, estas habilidades van orientadas hacia las técnicas de improvisación musical para crear y arreglar obras musicales con fines específicos. Estos fines son los de componer $y$ arreglar canciones para los diferentes eventos de las instituciones educativos y para ello es preciso desarrollar y profundizar en las herramientas que conjuntamente con la creatividad permitan la realización de obras musicales.

Cabe destacar que dentro de la UPEL se forman pedagogos del área musical y no músicos, sin embargo, se contempla dentro 
de los cursos de armonía (Armonía I y Armonía II) iniciar a los futuros docentes en esta área, por lo tanto hay que incentivar el desarrollo de las ideas musicales para la composición y el arreglo musical en ellos.

En la formación del docente en la UPEL, se debe tomar en cuenta un conjunto de habilidades que se construyen durante la estadía en la academia, estas habilidades, en el caso de los especialistas en música, son adquiridas partiendo de unas competencias mínimas de ingreso, donde el aspirante demuestre su inclinación hacia el arte de la música; en la ejecución de algún instrumento musical, destreza para el canto y capacidad para discriminar los diferentes instrumentos y sonidos presentes en una obra musical, y por otro lado debe manifestar valores éticos con una inclinación hacia la docencia.

Vargas (como se citó en García, 2009) señala que el objetivo de quien egresa de la mención Música, es desempeñarse como profesor de música a nivel escolar (desde preescolar hasta educación media y diversificada), aunque esto contradice lo expuesto en el Documento Base del Currículo (UPEL, 2011). Allí se manifiesta que el profesional está en capacidad de cubrir las exigencias de cualquier nivel donde se necesite a un docente en el área musical, también se plantea que el docente egresado de la especialidad de música no tendría la preparación suficiente para enseñar en una escuela de música, a menos que sea para enseñar niveles elementales de teoría y solfeo para niños así como de iniciación musical.

Además, como se ha señalado, a los docentes especialistas en música se les exige componer o arreglar una obra, el montaje de esta en diferentes eventos organizados por las instituciones donde laboran u organismo que dependen de las instituciones, y también estar capacitado en el oficio de director musical (director de coro, estudiantina y otros grupos), adicionalmente debe ser un virtuoso en la ejecución de algún instrumento y si son varios es mejor. Pero todos estos oficios de un profesional de la música, realizados en un conservatorio, toman alrededor de unos 15 a 17 años de estudio para tener una acreditación en las diferentes especialidades. Estas especialidades son: composición, director de orquesta, director de coro y maestro ejecutante de algún instrumento musical, pero en la UPEL se pretende formar un profesional en esta área con dos cursos del componente especializado dictados durante los cinco años de su carrera universitaria y en esta nueva transformación curricular que vive la UPEL solo en cuatro años.

Vale destacar que el investigador tiene más de 25 años de experiencia laboral en el área de la Educación Musical. Ha trabajado en diferentes escenarios educativos donde se imparte la educación musical, experiencia que dio aportes a la sustentación teórica de la investigación, también se ha desarrollado como músico en agrupaciones bajo el rol de arreglista, compositor, instrumentista y cantante, aunque últimamente se ha dedicado a la investigación y a la docencia musical, dejando un poco su labor artística, ya que su formación inicial fue como músico profesional dentro del sistema de escuelas de música. La doble experiencia profesional del investigador como músico y formador de formadores en Educación Musical, lo proveen de información válida y confiable para la determinación de la problemática de la investigación.

Todo lo anterior permitió formular el objetivo general de la presente investigación, el cual es generar un modelo teórico para el desarrollo de competencias relacionadas con el arreglo musical, que permita a los estudiantes de la especialidad Educación Musical de la Universidad Pedagógica Experimental Libertador, UPEL, Venezuela, 
un mejor desempeño durante su formación y en el campo laboral. Para alcanzar esto se describieron e interpretaron las percepciones de los actores institucionales con el fin de develar los criterios que sustentarían dicho modelo.

\section{MÉTODO}

Esta investigación es cualitativa; debido a la complejidad para evidenciar las relaciones humanas, las cuales no pueden ser tratadas tomando en cuenta el contexto en el que se desarrollan como las interpretaciones y significados que éstas adquieren para los sujetos.

Según Rodríguez (2000) la investigación cualitativa devela los significados que los humanos le dan a las acciones que desarrollan, con una finalidad no solamente descriptiva sino con la intención de proponer alternativas para su mejora.

Se utilizó la teoría fundamentada por ser una metodología que permitió indagar sobre lo desconocido para aportar conocimientos exhaustivos de una realidad específica (Strauss y Corbin, 1998), la cual se refiere al área de la armonía y del arreglo musical. Para ello se seleccionaron informantes claves, a partir de criterios específicos que se señalan a continuación:

\section{Docentes:}

- Pertenecientes a la universidad con experiencia en área de arreglo musical y que trabajaron en el sistema de educación venezolano convencional y no convencional

- Que manejara información relacionada con las exigencias del campo laboral para el profesional de la música y el docente en educación musical.

- Profesionales de la composición musical, es decir, músicos que ejercen como profesión la composición musical, y docentes que estén elaborando arreglos con fines específicos.

\section{Estudiantes de pregrado de la especialidad de música:}

- Estudiantes regulares de la especialidad educación musical y que ejerzan la docencia dentro del sistema de educación venezolano convencional y no convencional.

- Estudiantes de los últimos semestres de la especialidad educación musical por lo tanto puede expresar sus habilidades en el área de la armonía.

\section{Estudiantes de música}

- Estudiantes regulares de algunos de los sistemas no convencionales de formadores de músico (escuela de música, conservatorios y sistema de orquesta).

- Estos estudiantes estaban trabajando en el área de elaboración de arreglos musicales y hayan cursado los tres años armonías por lo menos.

\section{Profesores no graduados (docentes) que laboren en el área musical}

- Docente no graduados que laboran dentro sistema de educación venezolano convencional. 
Las técnicas e instrumentos empleados para la recolección de la información de la investigación se presentan en la Tabla 1.

Tabla 1. Técnicas e instrumento de recolección de información

\begin{tabular}{ll}
\hline Técnica & Instrumento \\
\hline Entrevista a profundidad & Guion de entrevistas \\
Observación participante & Registros \\
Análisis de contenido & Matriz de análisis \\
\hline
\end{tabular}

En cuanto al procedimiento utilizado este se basó en un estudio de campo, el cual permitió que se aplicara la entrevista a profundidad, a partir del guion que se elaboró con los insumos de la revisión bibliográfica. Los pasos que se llevaron a cabo se señalan a continuación:

- Se transcribieron las entrevistas.

- Se utilizó el programa Atlas-ti.

- Se analizaron los hallazgos para establecer una primera interpretación (codificación abierta) y así la denominación de los códigos (conceptos) a los elementos que iban surgiendo.

- Se agruparon los códigos por semejanzas o por estar relacionados (codificación axial) lo que conformó las categorías.

- Se contrastaron con los planteamientos teóricos relacionados (codificación selectiva) con la finalidad de enriquecer lo obtenido.

- Se integraron los elementos y se obtuvo la información de la realidad cómo es vista, que le dio marco a los aportes de esta investigación lo que estableció la columna vertebral de esta y permitió que se colocaran las bases en la construcción del modelo teórico para la formación del docente de música sustentado en el desarrollo de habilidades para el arreglo musical
El escenario donde se desarrolló la investigación fue dentro de la UPEL con las diferentes sedes que imparten la especialidad Educación Musical siendo estas cinco de las ocho que tiene la universidad siendo el Pedagógico de Miranda la sede donde se desempeña el investigador como profesor de armonía, así también el sistemas de escuelas de músicas donde se han formados los músicos más representativos de la música académica y popular de Venezuela, y en el sistema de orquesta que en la actualidad tienen una responsabilidad social de masificar la música, por lo tanto son los entes empleadores conjuntamente con el ministerio de educación cultura y deporte, de los profesores que gradúan la UPEL.

García (2009) señala que desde 1983, comenzó a impartirse la mención Música en la UPEL, específicamente en el Instituto Pedagógico de Caracas (IPC). A partir de 1993 en el Instituto Pedagógico de Maracay (IPMAR) y en 1996 en el Instituto Pedagógico de Miranda "José Manuel Siso Martínez" (IPMJMSM). En 1997, se hizo una revisión del diseño curricular y se estableció que el título que otorga dicha institución a partir de ese año es el de "Profesor de Educación Musical" equivalente a licenciado. 
RESULTADOS

Durante el proceso de análisis de las entrevistas se observó que los argumentos expuestos por los informantes claves fueron coincidiendo hasta la saturación total; lo que arrojó un área temática identificada como "el perfil laboral del profesional de la educación musical", desde las perspectivas de sus capacidades y competencias, asimismo surgieron unas categorías con sus respectivas subcategorías. (Ver Tabla 2)

Tabla 2. Categorías, subcategorías y elementos de la investigación

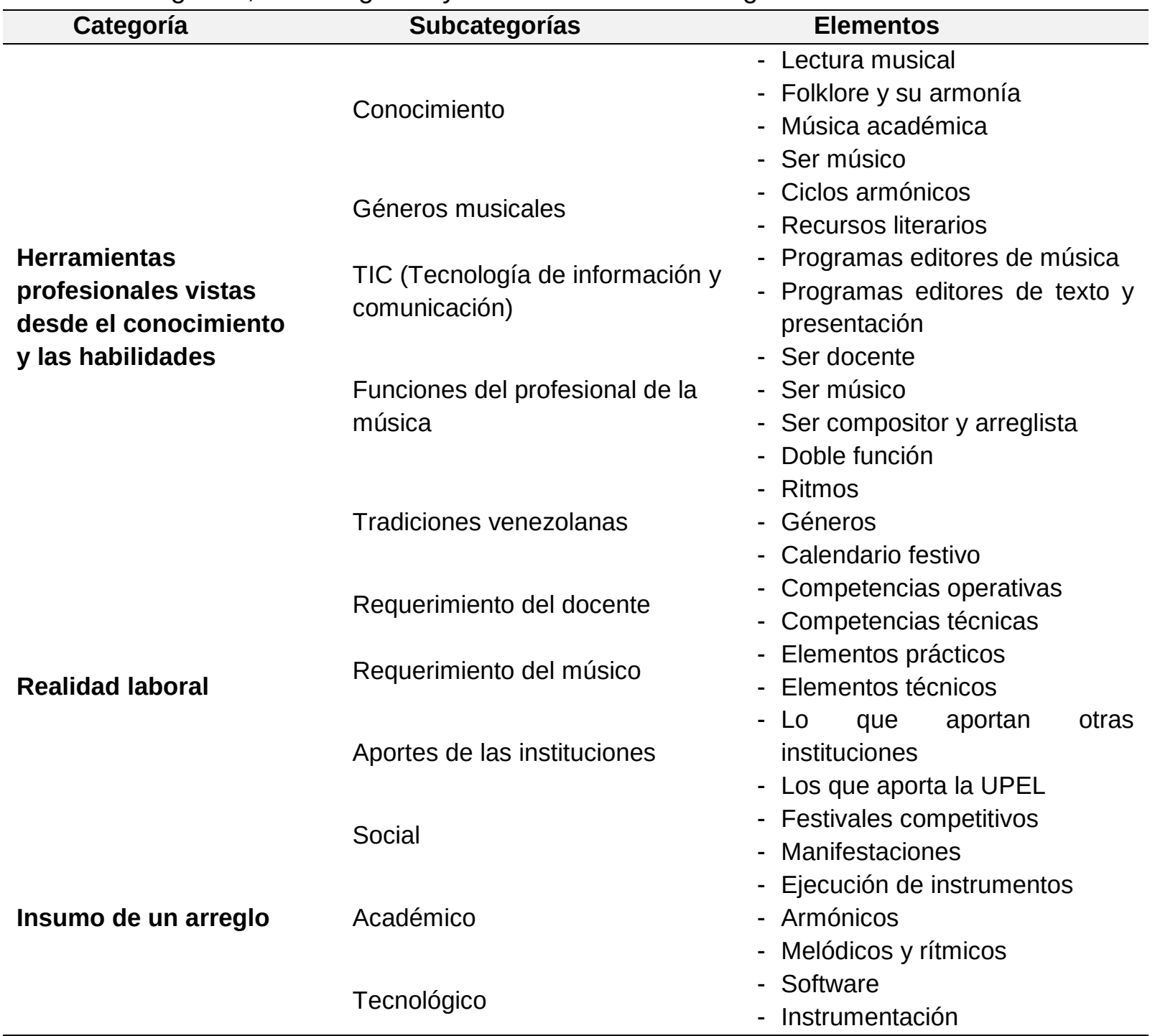

Luego, a partir de las subcategorías se fueron evidenciando elementos que permitieron generar el modelo, el cual se fue estructurando de acuerdo con la forma de una colmena. De allí que se haya denominado el modelo de la colmena, el cual está constituido por cavidades hexagonales que se van compactando por niveles, entre dos y cuatro.
La colmena se fue construyendo desde el centro hacia afuera. El tema central de la investigación representa su eje central, el cual es el "perfil del profesional de la enseñanza de la música", este refleja las habilidades que deben desarrollar los docentes del área de la especialidad de música durante su formación, con el fin de poder enfrentar las exigencias 
del campo laboral. Posteriormente, se fueron incorporando alrededor de ese eje, lo que sería otro nivel, las tres categorías que emergieron de la investigación: "herramientas profesionales visto desde el conocimiento $\mathrm{y}$ las habilidades", "realidad laboral" e "insumo de un arreglo", las cuales fueron identificadas con unas flechas que apuntan hacia arriba. Luego se conformó otro nivel en el que se colocaron las respectivas subcategorías y finalmente los elementos que iban derivándose de cada una de ellas. (Ver Gráfico 1)

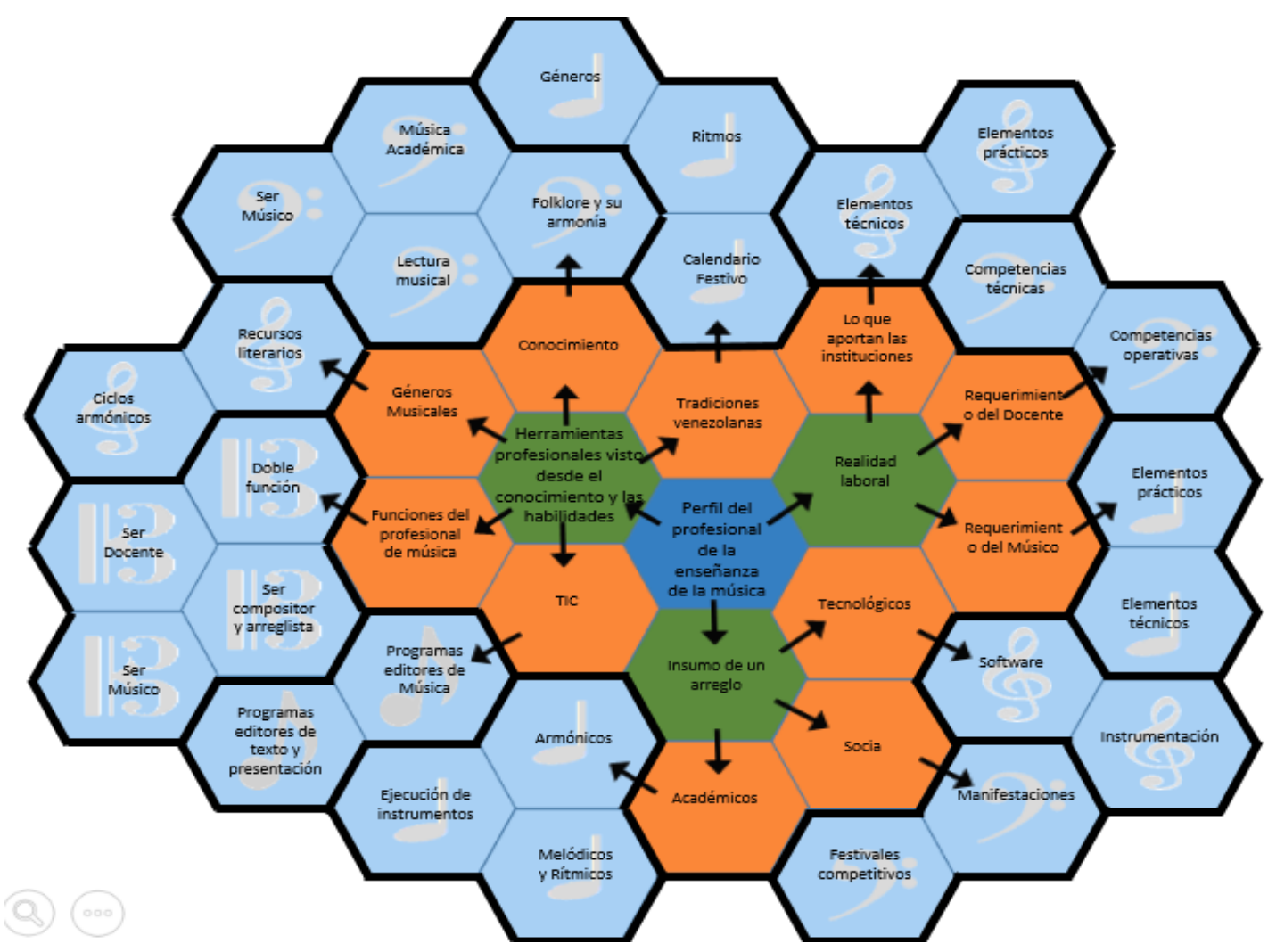

Gráfico 1. Modelo de la colmena. (Fuente: elaborado por el autor)

El perfil profesional es trabajado desde las competencias y habilidades profesionales que debe manejar aquella persona egresada de la UPEL en la especialidad de Educación Musical, para enfrentar el campo laboral de manera eficiente y sin inconvenientes en el aspecto específico de su formación docentemusical.

\section{Modelo teórico}

Como ya se señaló, el modelo generado se representó a partir de la imagen de una colmena de abeja, esto se debe a tres razones, la primera, desde la perspectiva de la acción pedagógica, la segunda, desde el sentido de pertenencia y la tercera, desde la epistemología de la palabra. (Ver Gráfico 2) 


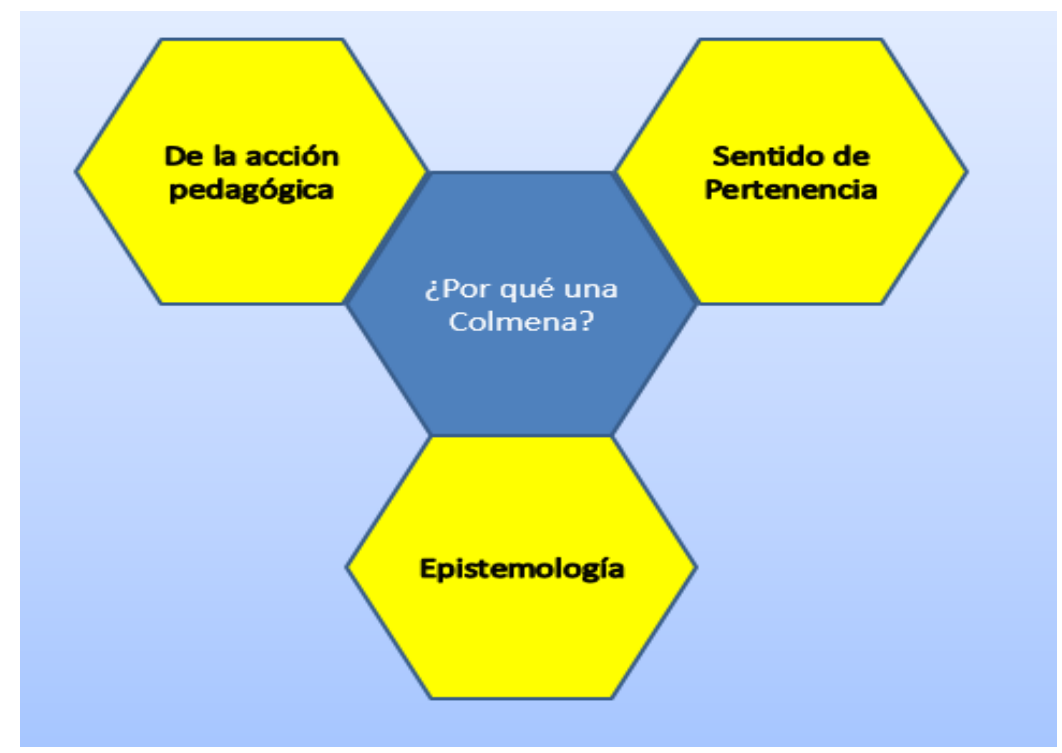

Gráfico 2. Origen de la representación gráfica del modelo. (Fuente: elaborado por el autor)

Desde la perspectiva de la acción pedagógica, la interpretación de lo expuesto por los entrevistados, la experiencia del investigador y la documentación permitieron construir el modelo que se asemeja a una colmena de abejas, producto de la metodología utilizada por el investigador en la acción pedagógica de los cursos de "Armonía I", "Armonía II" y "Arreglo y Creatividad". Allí los estudiantes comentaban que al representar en el pizarrón los acordes que se utilizaban en dichos cursos estos parecían un enlace para unir los elementos químicos de un compuesto dado o la celda de una colmena de abeja.

Desde el sentido de pertenencia, el logo de la institución en la que se realizó la investigación es una colmena (ver Gráfico 3). Según el artista plástico Luis Domingo Salazar, autor del emblema, señaló, en una entrevista publicada en la Revista UPEL Cultura No. 2 del año 2000, lo siguiente:

En mi emblema del Pedagógico Siso Martínez, la colmena está vista por dentro, con un corazón blanco que simboliza la luz en medio de la oscuridad del lugar donde están las abejas. Acaso por aquello de que estas casas nuestras de estudios ahuyentan las sombras. O porque son la manifestación de la moralidad y la intelectualidad. De la energía creadora, de la energía cósmica. (s.n)

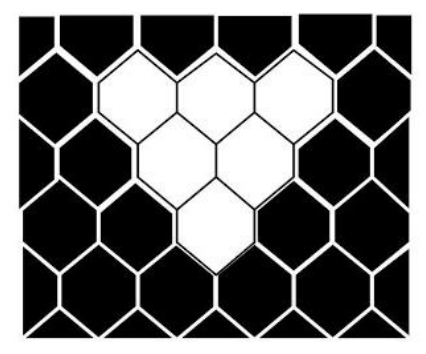

Gráfico 3. Logo del Instituto Pedagógico de Miranda José Manuel Siso Martínez, UPEL 
Para el modelo utilizado en esta investigación, la colmena representa una estructura rígida donde se relaciona las habilidades y competencias que debe manejar un profesional de la educación musical y que concuerden con una realidad laboral existe en la Venezuela actual.

Desde la epistemología de la palabra, los panales de abejas se estructuran como un esqueleto rígido que a su vez va formando concavidades en forma de celdillas hexagonales opuestas que comparten caras entre sus paredes y el fondo. Cuando se unen todas estas celdillas se origina el panal y son aprovechadas para incubar los huevos y posterior a su nacimiento proteger las crías, así mismo funciona como despensa para guardar sus alimentos y para luego ser digeridos en forma de pan de abejas, como también le da rigidez a la estructura. Para el modelo propuesto, la colmena representa una estructura rígida donde se relaciona las habilidades y competencias que debe manejar un profesional de la educación musical y que concuerden con una realidad laboral existe en la Venezuela actual.

\section{La perspectiva}

En la construcción de este modelo teórico surgieron cuatro niveles que componen la estructura completa de la colmena. Dicha estructura permite evidenciar la manera en la que se puede resolver el problema de las competencias del profesional de la educación musical que forma la UPEL para que pueda enfrentar efectivamente su quehacer docente. (Ver Gráfico 4)

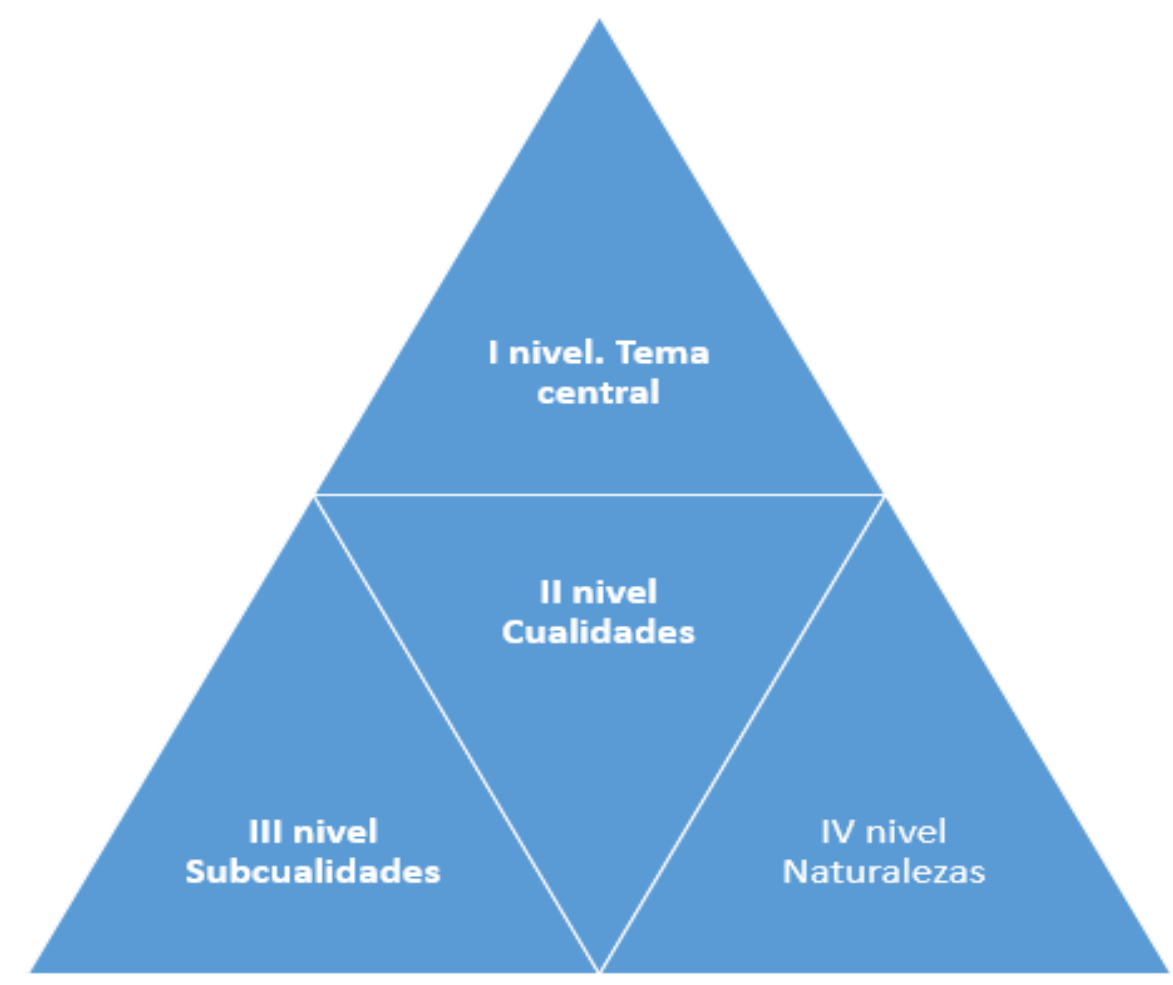

Gráfico 4. Niveles que componen la estructura de la colmena. (Fuente: elaborado por el autor) 
I nivel. Tema central. El tema central es el perfil del profesional de la enseñanza de la música, que debe ser ambicioso y denso, debido a las exigencias del campo laboral actual, el cual requiere un profesional de la docencia y al mismo tiempo de la música. En estos momentos la política de la universidad es formar docentes pero no el de formar músicos, escenario que está perjudicando a los egresados de la UPEL, porque no cumplen con las competencias y habilidades exigidos por los entes empleadores. Este problema debe solucionarse cambiando el currículo de la universidad donde se le dé igual importancia a la formación docente como musical de los futuros egresados como también buscar la manera de que el perfil de ingreso a la especialidad de educación musical tenga otro nivel, es decir que las competencias y habilidades con respecto al área musical de los que ingresan sean homogéneas y de más alto el nivel de conocimiento.

II nivel. Cualidades. Las cualidades de este modelo se refieren a las categorías ya señaladas (ver Tabla 2). Las cualidades constituyen aquellos rasgos que ese profesional especialista en la educación musical debe reflejar en sus destrezas y habilidades.

III nivel. Subcualidades. Las subcualidades permiten explicar las cualidades de forma general, es decir en este punto explicamos las partes que componen cada cualidad del modelo con detalles que permiten clarificar la denominación de la misma. Esto se refiere a las subcategorías. (ver Tabla 2)

IV nivel. Naturaleza. La naturaleza es la relación entre las categorías, subcategorías y elementos derivados de estas últimas. Por consiguiente este nivel presentó los insumos para la formulación de las competencias o habilidades relacionadas con el arreglo música. (ver Tabla 2)

\section{Competencias para el desarrollo del arreglo musical.}

A continuación se presentan las competencias que se derivaron al relacionar cualidad (categoría)/subcualidad (subcategoría)/naturaleza (elementos).

Todo docente de música debe manejar las siguientes competencias en relación con:

\section{Herramientas profesionales I subcualidad conocimiento I lectura musical}

- Conocer la teoría de la música, considerando que en la UPEL se enseña lo elemental de la anotación musical, cuando la realidad exige dominio amplio de la teoría musical con respecto a la armonía y la composición (teoría y solfeo, historia de la música, estética, y análisis musical entre otros).

- Dominio de la reproducción sonora de lo escrito, porque la UPEL imparte solfeo introductorio, cuando el mercado de trabajo de nuestros egresados exige dominar el solfeo en su plenitud (solfeo con y sin preparación de intervalos, y en diferentes tonalidades).

- Dominio de la correcta escritura de la música desde la copia y el dictado de la reproducción musical, son exigencia del mercado laboral, mientras en la UPEL no se aborda en ninguno de los cursos de la especialidad.

\section{Herramientas profesionales I subcualidad conocimiento / folklore y su armonía}

- Conocer las secuencias armónicas de los géneros musicales representativos de las manifestaciones culturales de 
Venezuela, debido a que el mercado laboral pretende un profesional que sea capaz de organizar eventos representativos culturales donde se realcen los valores culturales nacionales y regionales, mientras estos contenidos se abordan de forma superficial en un solo curso de la especialidad "antropología cultural".

- Dominar las diferentes perspectivas de la notación musical por cifrados alfanumérico y por cifrado de grados para que los estudiantes comiencen a tener nociones de las funciones armónicas, en el quehacer de un profesional de la música este conocimiento y aplicación es de gran utilidad para facilitar la compresión de los ciclos armónicos y a su vez la aplicación al momento de elaborar arreglos musicales para estos géneros, en los cursos de instrumentos impartidos en la UPEL se abordan un solo tipo de nomenclatura de cifrado que encierran a los estudiantes a no comprender la tonalidad porque se aborda en transferir un símbolo que representa un acorde, pero no se maneja su función armónica en el mundo tonal de la música.

\section{Herramientas profesionales I subcualidad conocimiento I música académica}

- Entender la estructura rítmica armónica y melódica de la música compuesta en Venezuela de orden académico y su evolución histórica dentro la corriente nacionalista utilizada hoy en día dentro del sistema de orquesta nacional como también aquellas proveniente de los géneros venezolanos populares, en el campo laboral los profesionales de la educación musical se les exige montar repertorio de corte académico venezolano dentro de las instituciones encargadas de la formación de músicos donde también se insertan los egresados de la UPEL, en los cursos de la especialidad de música son pocos los cursos y profesores que abordan estos géneros con fines de entender su estructura.

\section{Herramientas profesionales I subcualidad conocimiento I ser músico}

- Conocer el lenguaje técnico académico de la música para desarrollar los conceptos relevantes de los cursos de la especialidad a nivel teórico - práctico así como aquellos utilizados a niveles no tan formal para comprender a todos aquellos músicos que están inmerso dentro del campo laboral, la escuelas requieren de un docente capaz de corregir y expresar sus ideas claras con un lenguaje apropiado para cualquier nivel educativo, dentro de los cursos de la especialidad se manejan términos inadecuados por ser utilizados dentro de los argot de los músicos no estudiados pero se debe entender que la universidad pertenece a la academia y se debe utilizar un lenguaje apropiado para su nivel.

\section{Herramientas profesionales I subcualidad géneros musicales I ciclos armónicos}

- Determinar diferentes ciclos armónicos utilizados en los géneros de joropo venezolanos (Joropos a $3 / 4$ y 6/8) como los ciclos comunes de los valses, merengue venezolano, gaita, entre otros.

- Identificar los géneros con ciclos armónicos en tonalidad mayor y menor, asimismo los patrones rítmicos que los acompañan por región. 


\section{Herramientas \\ profesionales subcualidad géneros musicales I recursos literarios}

- Dominar la estructura de los versos, tipos y métrica española para la composición literaria de los géneros musicales venezolanos como también aquellos que utilizan versos libres. En el campo laboral se les exige a los profesionales de la música que compongan canciones que contenga un determinado mensaje en sus letras por lo tanto se debe manejar muy bien la estructura de los versos por géneros. En la UPEL y otras instituciones encargadas de formar profesionales dedicada a esta área de la música no toman en cuenta este contenido dentro de su currículo o plan de estudio.

\section{Herramientas profesionales I subcualidad TIC I programas editores de música}

- Dominar software o programas de edición musical que contemple tanto la edición de partituras que permite escuchar lo que se escribe en tiempo real es decir simultáneamente y también editores de grabaciones de audio que te permita grabar y editar una obra musical para mejorar su calidad dando así al profesional de la docencia una herramienta para utilizar en los montajes de su producto final, en los cursos ofrecidos en la especialidad educación musical de la UPEL solo abarcan software con edición de partituras. El campo laboral de la actualidad exige que grabes algunos de los trabajos para dar a conocer lo que se hace y presentar agrupaciones en salas de concierto importantes.

\section{Herramientas profesionales I subcualidad TIC I programas editores de texto y presentación}

- Dominar el uso de herramientas officemática en especial los editores de texto, hoja de cálculos y de presentación, para que de esta manera el profesional puede entregar todos sus trabajos con acabados profesional. En la UPEL específicamente a los estudiantes de la especialidad educación musical se les ofrece un curso optativo de informática aplicada a la educación donde se a barda el uso del paquete antes mencionado pero no son reforzados en los demás cursos.

\section{Herramientas profesionales I subcualidad funciones del profesor de música / ser músico}

- Ejecutar uno o varios instrumentos musicales incluyendo la voz cantada a nivel profesional o por lo menos medianamente aceptable, conociendo su técnica de ejecución y las limitantes que pueda presentar el instrumento como son el registro, digitación, colocación de las manos o apoyo de la columna de aire, entre otros. En los cursos ofertados a la especialidad educación musical se contempla en el de cuatro o instrumento I y otros de profundización, piano y técnica vocal. Estos cursos no son suficiente para desarrollar habilidades competentes en su ejecución, solo se conoce el instrumento superficialmente mientras en el campo laboral te exige que domine medianamente la ejecución de algún instrumento musical incluyendo las voz cantada. 


\section{Herramientas \\ profesionales subcualidad funciones del profesor de música / ser compositor y arreglista}

- Componer y arreglar obras musicales con diferentes instrumentaciones y distintos grados de dificultad en su ejecución, pensando en quienes serán sus músicos ejecutantes, es un aspecto que en la especialidad solo se aborda en tres cursos para desarrollar esta competencia que son los de "armonía I y II" y un optativo "arreglo y creatividad". En estos dos primeros hay docentes que lo plantea desde el enfoque de una armonía tradicional y su estudio contempla muchos años de estudio para culminar los mismos y solo se cuenta con dos periodos académicos para hacerlo. El campo de trabajo exige un docente que sea capaz de componer y arreglar obras musicales.

\section{Herramientas profesionales I subcualidad funciones del profesor de música / doble función}

- Dominar la ejecución por lo menos de dos instrumentos musicales al mismo tiempo, en la especialidad son pocos los cursos donde se trabaja esta integración y muchas veces depende más de la voluntad del facilitador que de las estrategias que se plantea en el curso, en el campo laboral se exige un docente que sea capaz de dirigir un coro y acompañarlo con algún otro instrumento musical este director además de dirigir que es una tarea difícil debe cantar y tocar un instrumento como el cuatro, la guitarra o el piano.

\section{Herramientas profesionales I subcualidad funciones del profesor de música / ser docente}

- Dominio de la acción docente visto desde la pedagogía, la planificación, la evaluación, la investigación y la elaboración de currículos orientados hacia la formación de las artes específicamente la de la música. En la UPEL se desarrollan estas competencias aunque existen docentes que manifiestan que hay muchas debilidades. Sin embargo, dicha universidad pedagógica es la única que ofrece un título de docente especialista en el área musical abalado por el Ministerio de Educación, y el campo laboral exige un profesional en la docencia que maneje lo expuesta anteriormente y con competencias musicales.

\section{Herramientas profesionales I subcualidad tradiciones venezolanas I ritmo \\ - Dominar los diferentes esquemas} rítmicos o patrones rítmicos en la ejecución de los instrumentos musicales partiendo desde instrumentos de percusión hasta los instrumentos armónicos utilizados en las manifestaciones tradicionales venezolanas como también los diferentes géneros propios de la música del folklore venezolano, en los cursos de la especialidad educación musical de la UPEL son pocos los que abordan estos contenidos y no se pone en manifiesto su práctica sino simplemente se abordan algunos a nivel teórico, en la realidad laboral se le exige al docente especialista a poner en escena algunas de las manifestaciones regionales dependiendo de la localidad de las instituciones. 


\section{Herramientas \\ profesionales subcualidad tradiciones venezolanas Igéneros}

- Dominar las diferentes secuencias armónicas pertenecientes a los géneros musicales venezolanos ya establecidos, como también giros armónicos más relevantes en la música popular venezolana perteneciente al folklore. En los cursos de la especialidad educación musical, donde se desarrollan estas habilidades en los estudiantes, simplemente no se abordan o el tiempo no es suficiente para trabajarlo. Esto se debe a que hay que solventar problemas de formación académica, para el graduado es importante conocer y ejecutar los diferentes géneros musicales porque se les exige en su lugar de trabajo el montaje de obras venezolanas y así poder representar a la institución en eventos.

\section{Herramientas profesionales I subcualidad tradiciones venezolanas I calendario festivo}

- Fortalecer en los estudiantes de la especialidad educación musical el estudio y el trabajo de campo sobre las tradiciones venezolanas y su sincretismo en Venezuela de las culturas que convergieron en la época de la conquista española que en la actualidad se pone en manifiesto en todo el territorio nacional. En la UPEL, dentro del programa del curso "historia de la música en Venezuela," es donde se trata este contenido pero no se hace transferencia de ello a los demás cursos, por lo tanto es muy poco lo que se aprende de este sincretismo. En el mercado laboral es imperante que el profesional maneje esta información debido a que en su trabajo les van a exigir la puesta en escena de estas manifestaciones.

\section{Realidad laboral I subcualidad requerimiento del docente I competencias técnicas}

- Desarrollar las competencias en cuanto se refiere a los detalles administrativos de la docencia como la planificación, evaluación y didáctica de la acción docente. Dentro la UPEL se desarrollan estas competencias aunque solo existen tres cursos de la especialidad que abordan los métodos de la enseñanza de la música y ninguno de ellos es relacionado a nuestro contexto social venezolano así mismo cuando aplican estrategias evaluativas parece un misterio el hecho de evaluar por competencias, siendo esta la mejor forma de evaluar el aprendizaje en las artes. En el mercado laboral al profesional de la docencia se les exige competencias del que hacer docente, en planificación por proyectos, evaluación del mismo y esto lleva inmerso las estrategias utilizadas para enseñar la música.

\section{Realidad laboral I subcualidad requerimiento del docente I competencias operativas}

- Aplicar de manera práctica todo lo referente a los métodos de enseñanza de la música transferido a nuestra realidad social, estrategias y dinámicas en la acción docente específicamente. En la UPEL es poca la oportunidad que tienen los estudiantes de aplicar estas estrategias y técnicas a la realidad, ya que el curso sobre métodos se comienza a ver después del quinto semestre. En el campo laboral es importante la práctica de los métodos porque permite lograr metas en menos tiempo con los estudiantes. 
Realidad laboral I subcualidad requerimiento del músico / elementos técnicas

- Desarrollar habilidades en los estudiantes de la especialidad educación musical netamente en el uso correcto del lenguaje técnico de la música como también las diferentes técnicas de lectura musical y la ejecución de instrumentos musicales de más fácil adquisición de la población, en la UPEL siempre se ha dicho que no se forman músicos en ella pero la realidad dice que un docente no puede enseñar lo que no sabe, así pues esta es una debilidad no formar músicos. En el campo laboral se les exige al docente del área de la música cumplir funciones del músico como el hecho de arreglar o componer una obra, formar una estudiantina, coro o grupo musical que son oficios del músico profesional.

\section{Realidad laboral I subcualidad requerimiento del músico / elementos prácticos}

- Elaborar y ejecutar de manera competente obras musicales, arreglos musicales y la dirección de grupos musicales con técnicas eficientes, eficaz y efectivas donde se evidencie en dominio de ellas en la práctica, en la UPEL los cursos relacionados con el desarrollo de estas competencias son mínimos y por separados y es difícil ver la integración y prosecución de las habilidades en la formación del estudiante; estos cursos son los de instrumentos, armonías y dirección coral, en el campo laboral te exigen estas competencias sin importar como la hagas pero tienes que hacerlas aunque debas pagar para poder obtener algo referentes del producto final.
Realidad laboral / subcualidad lo que aportan las instituciones /lo que aporta la UPEL

- Desarrollar habilidades en el docente en formación en la especialidad educación musical desde la pedagogía, la didáctica, y la formación de área del conocimiento, la UPEL es una institución que forma docentes capaces de enfrentar cualquier nivel educativo. Su debilidad está en el área de la especialidad donde se requiere formar un músico. Hay que desarrollar ambas competencias, porque el ente empleador requiere a un profesional docente y músico.

Realidad laboral / subcualidad lo que aportan las instituciones I lo que aportan las otras instituciones

- Crear convenios institucionales con las instituciones encargadas de formar profesionales de la música, para que desde el inicio de la formación académica del estudiante de educación musical se enfrente a la realidad laboral y así convertir las debilidades en fortaleza. En la UPEL no existe ningún convenio con otras instituciones encargadas de la formación académica de los músicos, y este el mayor nivel de exigencia que puede enfrentar el profesional de la educación musical (trabajar en las escuelas de música)

\section{Insumo de un arreglo I subcualidad social / manifestaciones}

- Desarrollar habilidades en la elaboración de arreglos musicales utilizando información proveniente de las manifestaciones culturales. En la UPEL existe un curso obligatorio como el de "historia de la música en Venezuela" donde 
se abordan estos temas, también hay dos optativos: "música y artes escénicas" y "antropología cultural". En el mercado laboral se les exige al profesional de la docencia de la música que monte eventos relacionados con el calendario festivo como son los de San Pedro, Cruz de mayo, entre otros, y este requiere no solo conocimiento sobre las festividades sino el del quehacer del músico profesional.

\section{Insumo de un arreglo I subcualidad social / festivales competitivos}

- Desarrollar habilidades en la composición y elaboración de arreglos musicales utilizando las normas y estatutos de los festivales más destacados a nivel estudiantil o dentro del ambiente laboral. En la UPEL no hay cursos relacionados con la elaboración y organización de eventos competitivos, pero la universidad organiza un evento parecido a los que se emprenden en estas instituciones como es el Festival de la voz UPEL en la sede Miranda, pero no pertenece al currículo y puede participar cualquier miembro de la comunidad.

\section{Insumo de un arreglo I subcualidad académico / armónicos}

- Conocer por lo menos un tratado para la elaboración de arreglos musicales basados en la armonía funcional moderna donde el profesional pueda desarrollar competencias en poco tiempo y al practicar el oficio pueda profundizar a través de la investigación el perfeccionamiento de las técnicas de la armonización con propósitos específicos. En la UPEL se pretende dar la armonía como se ha enseñado tradicionalmente en la escuela de música, esto requiere de mucho tiempo (años) de estudios para comprender la elaboración de arreglos partiendo de solo una línea melódica por lo tanto no es conveniente. El mercado laboral requiere que el profesional de la docencia musical elabore arreglos musicales partiendo de solo una melodía que muchas veces tiene que transcribirlas de un audio a la partitura.

\section{Insumo de un arreglo I subcualidad académico / melódico y rítmico}

- Conocer sobre la estructura y elemento de la melodía y el ritmo como también su concordancia con la estructura rítmica de la métrica española en idioma del castellano. En la UPEL, al igual que los demás instituciones encargadas de formar músicos compositores y arreglistas, no contemplan este contenido tan importante para la elaboración de una obra musical con letra y arreglos musicales donde se debe utilizar fragmentos coherentes de la poesía y así lograr un arreglo coral visto desde la literatura y la musicalización. En el campo laboral no encontramos obras ni arreglos con características específicas donde se refleje todo lo que exige el patrono, por lo tanto ese profesional de la música debe elaborarlo.

\section{Insumo de un arreglo I subcualidad académico I ejecución de instrumentos}

- Ejecutar y conocer a profundidad las técnicas de ejecución de algún instrumento musical; de ser posible uno de aquellos de más fácil adquisición como es el cuatro, la guitarra, la flauta dulce y el canto para que sirva de apoyo para la elaboración de arreglos musicales. En la UPEL se administran pocos cursos que desarrolle la ejecución instrumental; obligatorio solo tres piano I y II y cuatro donde se desarrolla 
pocos competencias en la ejecución instrumental, cuando el mercado laboral requiere un docente que ejecute varios instrumentos musicales entre ellos la voz cantado que a su vez son herramientas necesarias para la elaboración de arreglos musicales con fines específicos.

- Conocer la estructura y técnicas de los instrumentos más comunes de la cultura popular venezolana y de la orquesta para poder escribir arreglos sin inconvenientes técnicos de ejecución y registro. En la UPEL no se manejan contenidos y mucho menos curso de orquestación; donde se aborden los requerimientos técnicos y de ejecución de los diferentes instrumentos musicales. En el campo laboral se les exige al docente especialista en música elaborar arreglos para una variedad de formatos o integración de instrumentos en un grupo musical.

\section{Insumo de un arreglo I subcualidad tecnológico / instrumentación}

- Dominar la ejecución de un instrumento armónico - melódico que sirva de apoyo para interpretar la obra musical completa o fragmentos para resolver algún obstáculo que se presente en la elaboración de la misma, al igual que lo descrito en la naturaleza ejecución de instrumento donde se mencionó el conocer la ejecución técnica de ellos. En la UPEL tampoco se enseñan cuáles serían los formatos más comunes de la instrumentación que encontramos en la realidad laboral y que también suelen ser muy variados para la conformación de grupos musicales, cuando se refiere a la instrumentación es la conformación instrumental del grupo, en otras palabras los instrumentos que se ejecutan tomando en cuenta desde un cuatro, guitarra, percusión, hasta la voz o las voces humanas.

\section{Insumo de un arreglo I subcualidad tecnológico / software}

- Dominar el manejo de un software especializado para la edición de partituras que permita escuchar lo que se escribe en tiempo real y así tener una idea clara de cómo está quedando el arreglo musical o la composición de la obra musical, en la UPEL se administra el curso de informática aplicada a la música que contemple niveles básicos del manejo de un software editor de partituras, el mercado laboral te exige un que hagas el trabajo como sea pero este recurso facilita la elaboración de los arreglos musicales.

- Dominar la operatividad de software especializados de grabación y edición de audio que permita la posterior reproducción de sonido de las voces facilitando que los coros que no lean música puedan estudiar las piezas fácilmente, el elaborar un demo de la agrupación que dirija para ser utilizada de promoción, entre otras cosas. En la UPEL no se enseña el manejo de software especializado a la grabación y tampoco se cuenta con una plataforma tecnológica para hacer esto posible. En el mercado laboral se pide a los docentes especialistas que realice videos de las presentaciones, pero estos son exigencias totalmente fuera de las funciones del profesional de la docencia musical.

\section{CONCLUSIONES}

La voluntad política de la institución de asumir nuevos paradigmas en su estructura sistémica es un factor importante porque como es cierto que todo cambio genera una resistencia siempre se encontrarán algunos individuos que no quiere salir de su zona de seguridad, por lo tanto estos no querrán desaprender para luego aprender como formar docentes para un mercado laboral 
bastante exigente, lo mismo sucede con los encargados de hacer administrativamente los cambios.

Para la UPEL, este momento de transformación curricular donde los paradigmas del cómo se forma a los estudiantes y el cómo se debe administrar las unidades curriculares que actualmente se manejan como cursos y ante el cambio estructural del currículo de la universidad, es pertinente que para la especialidad de música se tomen las consideraciones del modelo del colmena emanado de este trabajo de investigación que nace por la preocupación del autor para mejorar la calidad profesional de los egresados de educación musical.

Así mismo, estimar el hecho de querer incluir muchos de los contenidos que desarrollarán las competencias de los docentes en formación en la especialidad educación musical, como ejes transversales que deben incluirse en todos los cursos de la especialidad, y de ser posible en los otros cursos que pertenezcan a los demás componentes. De esta manera se garantizaría la ilación de las áreas temáticas de la música y de la buena prosecución de la formación académica de ese futuro docente especialista en el área de la educación musical, que debe estar en concordancia con los estatutos legales donde se garantiza la formación de un profesional capaz de cumplir las exigencias expuestas por el Estado y el campo laboral.

Este modelo no solo pretende formar a un docente exclusivamente con competencias en el área de la música, sino que también debe formar un docente capaz de ser investigador, creativo, proactivo, como en todo lo que implica el quehacer docente, del músico y en las artes en general.

La profesión de la educación es una de las más importantes en la construcción de una sociedad y debería ser vista como la profesión de las profesiones, pero para lograr estos objetivos es preciso hacer valorar los títulos socialmente, y para ganar ese respeto la universidad debe formar cada día a mejores profesionales capaces de defender los derechos del educador desde el resultado de su trabajo, de aquí la reivindicación de sus sueldos, el equilibrio de quienes ejerzan nuestro profesión, ya que se encuentran muchas personas sin titularidad que se hacen llamar docentes.

El docente facilitador de la UPEL tiene el compromiso de desarrollar en los estudiantes de la especialidad educación musical destrezas en el área de la composición, arreglos musicales y dirección de grupos con niveles competitivos estándares que pueda competir con músicos profesionales, en otras palabras no que sea un virtuoso pero sí que pueda defenderse en el campo profesional, esto se puede lograr integrando en los cursos de la especialidad con aspectos teórico práctico del oficio y competencias del músico profesional desde el comienzo de la carrera hasta su culminación, para que de esta manera los actores involucrados en la formación de los futuros docentes trabajen con esmero en el desarrollo de las competencias musicales, y así lograr cumplir con las exigencias del estado en formar profesiones capaz de enfrentar los requerimientos laborales que pretende la sociedad.

\section{REFERENCIAS}

García F., Z. (2009). La Enseñanza de la Música en las Universidades de Venezuela. Docencia Universitaria, X(2). Recuperado de http://www.ucv.ve/fileadmin/user_upload /sadpro/Documentos/docencia_vol10_n2 _2009/4_Zaira_Garcia.pdf

Guzmán, B. y Rodríguez, R. (2012). Competencias de estudiantes que ingresan a Educación Musical. España: Editorial Académica Española 
Rodríguez, J. (2000). Investigación Cualitativa en Educación Musical: un nuevo reto en el contexto educativo español. Revista de la Lista Electrónica Europea de Música en la Educación. Recuperado de http://musica.rediris.es/leeme/revista/rod riguezja00.pdf

Strauss, A., y Corbin, J. (1998). Basics of qualitative research: Techniques and procedures for developing grounded theory (2nd ed.). Thousand Oaks, CA: Sage

Universidad Pedagógica Experimental Libertador [UPEL] (2011). Documento base del currículo UPEL. Recuperado de:

http://geminis.upel.edu.ve/vdoc/Reposito rio/TransformacionCurricular/Lineamient oS/DOCUMENTO_BASE_CURRICULO _UPEL.pdf 